Original Article

\title{
Role of Decompressive Craniectomy in severe Traumatic Brain Injury: An Institutional Experience
}

\author{
Imran Altaf
}

Department of Neurosurgery, Khawja Muhammad Safdar Medical College, Sialkot - Pakistan

\begin{abstract}
Objective: The role that Decompressive Craniectomy plays in managing patients suffering from traumatic brain injury still to date remains controversial, and whether it improves clinical outcomes in severe traumatic brain injury patients is still a matter of debate. The present study was designed to assess the outcome following the Decompressive Craniectomy procedures performed in our setup for patients presenting with severe traumatic brain injury.
\end{abstract}

Materials and Methods: This was a retrospective study wherein the medical records of adult patients that presented with an initial Glasgow Coma Scale (GCS) $\leq 8$ and in whom decompressive craniectomy had been carried out for severe traumatic brain injury were retrospectively analyzed. Patients in whom decompressive craniectomy had been carried out for causes other than trauma and patients with initial GCS $\geq 9$ were excluded from the study. The studied parameters included age, sex, initial GCS, computed tomography (CT) brain diagnosis, and the outcome according to the Glasgow coma outcome scale (GOS).

Results: The study included 12 patients, and of these 12 patients operated with Decompressive Craniectomy for severe traumatic brain injury only 2 survived. The mortality was $83.3 \%$. The initial GCS and age were not statistically different between the survivors and the non-survivors. Based on the Glasgow Outcome Scale (GOS) only 1 patient had a good outcome. Overall, an unfavorable outcome based on the GOS score was seen in $91.7 \%$ of patients.

Conclusion: Our study concludes that Decompressive Craniectomy is associated with high mortality in patients presenting with severe traumatic brain injury and does not seem to offer a better alternative to standard medical management.

Keywords: Decompressive Craniectomy, severe traumatic brain injury, Glasgow coma outcome scale.

Corresponding Author: Imran Altaf

Department of Neurosurgery, Khawja Muhammad Safdar

Medical College, Sialkot - Pakistan

Email: drimr2@hotmail.com

Date of Submission: 12-09-2021

Date of Revision: 09-11-2021

Date of Acceptance: 30-11-2021
Date of Online Publishing: 31-12-2021

Date of Print: 31-12-2021

DOI: $10.36552 /$ pjns.v25i4.611

\section{INTRODUCTION}

Severe traumatic brain injury (TBI) occurs in about 
$10 \%$ of the patients getting admitted to hospital after suffering TBI. $^{1}$ The mortality and morbidity are high and the percentage of patients that either die or survive with severe disability following severe traumatic brain injury is approximately $60 \%{ }^{2,3}$ The mortality and morbidity remains very high even in the USA and Europe and even in centers that specialize in neurotrauma management and reaches upto atleast $50 \%$ even in such specialized neurotrauma centers. ${ }^{1}$ The burden of severe traumatic brain injury is biggest in low and middle-income countries. ${ }^{1,2}$ Further compounding this factor is the fact that in low and middle-income countries the mortality was found to be $21 \%$ higher in severe $\mathrm{TBI}$ patients when compared with highincome countries. ${ }^{1}$

Decompressive craniectomy (DC) is a widely used and well-established procedure for managing patients presenting with traumatic brain injury. ${ }^{4-8}$ The procedure, however, still to date remains controversial and the role it plays in managing traumatic brain injury patients is still debated. 2,3,5,7-11 The procedure initially lost favor with the neurosurgeons after the poor results and very high mortality that showed-up in early studies. However, there was a resurgence in interest in DC after promising results showed up in later studies. ${ }^{1,7}$ Studies comparing DC with craniotomy in the management of traumatic brain injury have shown conflicting results with some studies finding DC to be associated with a better outcome and others finding DC to be associated with a worse prognosis. ${ }^{10,12-15}$ The two large decra and resuscip trial comparing DC with standard medical care in patients suffering from severe traumatic brain injury found that DC leads to more unfavorable outcome compared to standard medical management. ${ }^{2}$ Making the procedure more controversial is the perception that even if DC reduces mortality it does so only by increasing the number of patients in the vegetative or severely disabled state, thus again putting into question the rationality of the procedure. ${ }^{7,16-19}$ This has led to a scenario where a uniform protocol for performing the procedure does not exist and different neurosurgical centers and neurosurgeons have their own preferences and protocols. ${ }^{4,20}$ The present study had been designed for assessing the outcome and efficacy of the DC procedures performed in our setup for severe TBI patients.

\section{MATERIALS AND METHODS}

\section{Study Design and Setting}

This study was a retrospective observational study. It was conducted in the department of neurosurgery Allama lqbal Hospital Sialkot. Medical records of adult patients in whom decompressive craniectomy had been carried out for severe traumatic brain injury from February 2020 to June 2021 were retrospectively analyzed. The parameters studied included age, sex, initial GCS, CT brain diagnosis, and the clinical outcome according to the Glasgow coma outcome scale (GOS).As the study is retrospective in nature so ethical committee approval was not required.

\section{Inclusion Criteria}

All adult patients in whom decompressive craniectomy had been carried out for traumatic brain injury with initial GCS $\leq 8$ were included in the study. The radiological parameters for inclusion consisted of a CT brain showing either a (1) Acute subdural hematoma/hemorrhagic contusion with a midline shift $\geq 5 \mathrm{~mm}$ or (2) Diffuse brain swelling and basal cisternal effacement with clinical signs of herniation.

\section{Exclusion Criteria}

Patients in whom decompressive craniectomy had been carried out for causes other than trauma were excluded from the study. Patients with a follow-up period $<6$ months and patients with initial GCS $\geq 9$ were also excluded from the study. 


\section{Operative Technique and Management Protocol}

The decompressive craniectomy had been performed in one of the two ways. Either a burr hole craniotomy was done and the flap was not placed back at the end of the procedure or a large temporal craniectomy had been done upto the base of the skull. The dura was either left open at the end of the procedure or the edges approximated with loosely placed stitches. The follow-up of the surviving patients was for more than 3 months. The Glasgow Outcome Scale (GOS) was utilized for categorizing the outcome of the surviving patients. The outcome was classified as "favorable" if the GOS score was 4 or 5 , and was deemed "unfavorable" if the GOS score remained 3 or less.

\section{Data Analysis}

The surviving and the non-surviving patients were compared for the initial GCS and age using the independent t-test. The statistical significance for all analyses was determined at $\mathrm{P}<0.05$.

\section{RESULTS}

\section{Gender Distribution}

The study included twelve patients. There were 6 males and 6 females.

\section{Age Distribution}

The age range was 17 years to 50 years with a mean age of $32.5 \pm 10.94$ years.

\section{Operated Pathologies}

The operated pathologies were Acute Subdural hematoma in 7 patients, Acute subdural hematoma + large contusion in 3 patients, bilateral frontal contusions in 1 patient, and diffuse axonal injury + brain edema in 1 patient. In 11 patients an initial burr hole craniotomy had been carried out and the flap was not put back, and in 1 patient a craniectomy had been done.

\section{Outcome after Decompressive Craniectomy}

Of the 12 operated patients, only 2 survived. The mortality was $83.3 \%$. The initial GCS and age were not statistically different between the survivors and the patients that did not survive as shown in table 1.

Table 1: Comparison of initial GCS and age between the survivors and non-survivors.

$\begin{array}{lccc}\text { Parameters } & \text { Survivor } & \text { Non-survivor } & \text { P-value } \\ \begin{array}{l}\text { Age } \\ \text { Mean } \pm \text { SD (Years) }\end{array} & 23 \pm 5 & 34.4 \pm 10.82 & 0.1868 \\ \begin{array}{l}\text { Initial GCS } \\ \text { Mean } \pm \text { SD }\end{array} & 7.5 \pm 0.5 & 5.7 \pm 1.49 & 0.1326\end{array}$

Postoperatively no surgical complications e.g. wound infection, re-bleed, etc. occurred in any patient and none of the patients required reoperation. The time from surgery to death for the expired patients ranged between 23 hours to 21 days (mean $=7.9$ days)

The initial presenting GCS of the two surviving patients were 7 and 8 . One of them remains dependent and bedridden with GCS 10 and is fed with a nasogastric tube 3 months postoperatively. The second patient is fully functional and living a normal life 9 months postoperatively. Based on the Glasgow Outcome Scale (GOS score) only 1 patient had a good outcome. The $2^{\text {nd }}$ patient remains bedridden and dependent. Overall, unfavorable outcome based on the GOS score was seen in $91.7 \%$ of patients.

\section{DISCUSSION}

The history of DC for traumatic brain injury is a long and checkered one. ${ }^{7}$ Studies conducted for ascertaining the role that DC plays in managing 
TBI patients have explored two important aspects i.e. (1) Comparing decompressive craniectomy with craniotomy in the management of TBI and (2) Comparing decompressive craniectomy with standard medical care in managing TBI patients.

The topic of craniotomy or craniectomy for traumatic brain injury remains controversial and studies have shown contrasting results. ${ }^{10,12-15}$ However, the meta-analysis by Kevin Phan et al ${ }^{10}$ concluded that when performed for acute subdural hematoma, decompressive craniectomy was found to be associated with a worse clinical outcome as compared to craniotomy.

The landmark DECRA study based on the data from 2002 to 2010 comparing DC with standard medical management in traumatic brain injury found that unfavorable outcome was observed in $70 \%$ of the patients in whom DC had been carried out compared to an unfavorable outcome of $50 \%$ in the group of patients managed with conservative medical management. This landmark study concluded that standard medical management leads to a more favorable outcome when compared to decompressive craniectomy, and the healthcare system could save millions of dollars by employing standard medical management instead of surgical decompression., ${ }^{9,21}$ The meta-analysis by Fatima et $\mathrm{al}^{11}$ concluded that the clinical outcome remains the same in severe $\mathrm{TBI}$ patients whether decompressive craniectomy or conservative medical management was employed. The metaanalysis carried out by Wang et $\mathrm{al}^{22}$ also concluded that decompressive craniectomy did not improve outcomes compared to conservative management in severe TBI patients. Another point of concern that has been raised consistently is that although DC reduces mortality it does so only to increase the number of patients in the vegetative or severely disabled state. ${ }^{7,16-19}$

We had a mortality of $83.3 \%$ which although falls within the $13 \%-90 \%{ }^{7}$ mortality range described in literature yet is very high. Overall poor outcome was observed in $91.7 \%$ of patients.
Our study thus seems to lead to the conclusion that DC did not offer any benefit to these patients suffering from TBI compared to the scenario if they had been managed with standard medical management only, and seems to support the findings of the multiple studies ${ }^{2,} 9$ and meta analysis ${ }^{11,22}$ that found the results of decompressive craniectomy to be not significantly different from standard medical management.

\section{Limitations}

The study is a retrospective study conducted at a single center. Furthermore, it was nonrandomized and the patient population was small. A multi-center, randomized, prospective study with a large patient population is required to accurately define the role that Decompressive Craniectomy plays in the management of severe TBI patients.

\section{CONCLUSION}

Our study concludes that DC is associated with high mortality in patients presenting with severe traumatic brain injury and does not seem to offer a better alternative to standard medical management.

\section{REFERENCES}

1. Sahuquillo J, Dennis JA. Decompressive craniectomy for the treatment of high intracranial pressure in closed traumatic brain injury. Cochrane Database Syst Rev. 2019; 12 (12): CD003983.

2. Shah DB, Paudel P, Joshi S, Karki P, Sharma GR. Outcome of Decompressive Craniectomy for Traumatic Brain Injury: An Institutional-Based Analysis from Nepal. Asian J Neurosurg. 2021; 16 (2): 288-293.

3. Laghari AA, Bari ME, Waqas M, Ahmed SI, Nathani KR, Moazzam W. Outcome of Decompressive Craniectomy in Traumatic Closed Head Injury. Asian J Neurosurg. 2018; 13 (4): 1053-1056.

4. Kwon YS, Yang $\mathrm{KH}$, Lee $\mathrm{YH}$. Craniotomy or Decompressive Craniectomy for Acute Subdural 
Hematomas: Surgical Selection and Clinical Outcome. Korean J Neurotrauma. 2016; 12 (1): 227.

5. Winter $C D$, Adamides A, Rosenfeld JV. The role of decompressive craniectomy in the management of traumatic brain injury: a critical review. J Clin Neurosci. 2005; 12 (6): 619-23.

6. NdiayeSy EHC, Cisse Y, Thiam AB, Barry LF, Mbaye $M$, Diop $A$, et al. Decompressive craniectomy: indications and results of 24 cases at the neurosurgery clinic of Fann university hospital of Dakar. Pan Afr Med J. 2021; 38: 399.

7. Mezue W, Ndubuisi C. Decompressive craniectomy in the management of traumatic brain injury: a review of current practice. Open Access Surgery, 2015; 8: 73-83.

8. Choudhary NK, Bhargava R. Decompressive Craniectomy in Diffuse Traumatic Brain Injury: An Industrial Hospital Study. Asian J Neurosurg. 2018; 13 (2): 314-318.

9. Cooper DJ, Rosenfeld JV, Murray L, Arabi YM, Davies AR, D'Urso $P$, et al. Decompressive craniectomy in diffuse traumatic brain injury. $\mathrm{N}$ Engl J Med. 2011; 364: 1493-502.

10. Phan K, Moore JM, Griessenauer C, Dmytriw AA, Scherman DB, Sheik-Ali $S$ et al. Craniotomy Versus Decompressive Craniectomy for Acute Subdural Hematoma: Systematic Review and Meta-Analysis. World Neurosurgery, 2017; 101: 677-685.e2.

11. Fatima N, Al Rumaihi G, Shuaib A, Saqqur M. The Role of Decompressive Craniectomy in Traumatic Brain Injury: A Systematic Review and Metaanalysis. Asian J Neurosurg. 2019; 14 (2): 371-381.

12. Coplin WM, Cullen NK, Policherla PN, Vinas FC, Wilseck JM, Zafonte RD, Rengachary SS. Safety and feasibility of craniectomy with duraplasty as the initial surgical intervention for severe traumatic brain injury. J Trauma. 2001; 50 (6): 1050-9.

13. Huang AP, Tu YK, Tsai YH, Chen YS, Hong WC, Yang CC, et al. Decompressive craniectomy as the primary surgical intervention for hemorrhagic contusion. J Neurotrauma, 2008; 25 (11): 1347-54.

14. Yılmaz I, Ertem DH, Kılıç $M$, Altaş K, Mirhasilova $M$, Özdemir B, et al. Factors associated with mortality in acute subdural hematoma: Is decompressive craniectomy effective? Ulus Travma Acil Cerrahi Derg. 2019; 25: 147-153.

15. Vilcinis R, Bunevicius A, Tamasauskas A. The Association of Surgical Method with Outcomes of Acute Subdural Hematoma Patients: Experience with 643 Consecutive Patients. World Neurosurg. 2017; 101: 335-342.

16. Honeybul S, Ho KM, Lind CRP, Gillett GR. The current role of decompressive craniectomy for severe traumatic brain injury. J Clin Neurosci. 2017; 43: 11-15.

17. Honeybul S. Long-term outcome following severe traumatic brain injury: ethical considerations. J Neurosurg Sci. 2018 Oct; 62 (5): 599-605.

18. Honeybul S, Ho KM, Gillett GR. Reconsidering the role of decompressive craniectomy for neurological emergencies. J Crit Care, 2017; 39: 185-189.

19. Honeybul S, Ho KM, Gillett GR. Long-Term outcome following decompressive craniectomy: An inconvenient truth? Current Opinion in Critical Care, 2018; 24 (2): 97-104.

20. Hartings JA, Vidgeon S, Strong AJ, Zacko C, Vagal A, Andaluz N, et al; Co-Operative Studies on Brain Injury Depolarizations. Surgical management of traumatic brain injury: a comparative-effectiveness study of 2 centers. J Neurosurg. 2014; 120 (2): 43446.

21. Honeybul S, Ho KM, Lind CR. What can be learned from the DECRA study. World Neurosurg. 2013; 79 (1): 159-61.

22. Wang JW, Li JP, Song YL, Tan K, Wang Y, Li T, et al. Decompressive craniectomy in neurocritical care. J Clin Neurosci. 2016; 27: 1-7. 


\section{Additional Information}

Disclosures: Authors report no conflict of interest.

Ethical Review Board Approval: As the study is retrospective in nature so ethical committee approval was not required.

Conflicts of Interest:

In compliance with the ICMJE uniform disclosure form, all authors declare the following:

Financial Relationships: All authors have declared that they have no financial relationships at present or within the previous three years with any organizations that might have an interest in the submitted work.

Other Relationships: All authors have declared that there are no other relationships or activities that could appear to have influenced the submitted work.

\section{AUTHORS CONTRIBUTIONS}

\begin{tabular}{|l|l|l|}
\hline Sr.\# & Author's Full Name & Intellectual Contribution to Paper in Terms of: \\
\hline 1. & Imran Altaf & $\begin{array}{l}\text { Study design, paper writing, data calculations, } \\
\text { literature review, resultsand methodology }\end{array}$ \\
\hline
\end{tabular}

